\title{
Gas sensor array based on multi-walled carbon nanotubes and polymer
}

\author{
Tuoyu Yang ${ }^{1, a}$, Zitao Li $^{1, b}$, Ying Dong ${ }^{1, c}$ and Xiaohao Wang ${ }^{1,2, d}$ \\ ${ }^{1}$ Graduate School at Shenzhen, Tsinghua University, University town of Shenzhen, Shenzhen \\ 518055, China \\ ${ }^{2}$ Tsinghua-Berkeley Shenzhen Institute, Tsinghua University, University Town of Shenzhen, \\ Shenzhen 518055, China

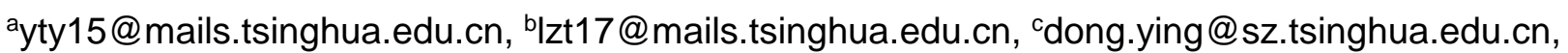 \\ dwang.xiaohao@sz.tsinghua.edu.cn
}

\section{Keywords: gas sensor array; VOCs; MWCNTs; polymer}

Abstract.The multi-walled carbon nanotubes combined with four polymers respectively were used as a sensitive layer of in the monolithically integrated gas sensor array. The multi-walled carbon nanotubes in the gas sensor array were added using a dip coating, and the corresponding polymer was added to different sensors in the array by spraying coating. The sensor array demonstrated the ability for detection and discrimination of different volatile organic compunds (VOCs). The adsorption characteristics of VOCs vary with different kinds of polymers. The adsorption of VOCs affected the conductive network in the sensitive layer and caused its resistance change. By measuring the change in resistance of these sensors as the output signal of the sensor array, the experimental results demonstrated the potential of using such devices for VOCs detection and analysis applications.

\section{Introduction}

The detection of VOCs concentration is becoming more and more widely used in application such as environmental monitoring, food quality inspection and medical examination [1-7], and the development of corresponding real-time detection equipment and IoT sensor nodes is becoming more and more important $[8,9]$. As a commonly used detection device, the core component of the electronic nose is a gas sensor array, which is composed of a set of gas sensors with different sensing characteristics. A commonly used gas sensor is typically a metal oxide semiconductor (MOS) type gas sensor, which is based on the oxidation-reduction reaction of the analyte with the surface oxygen ions [10]. It is difficult for MOS sensor to achieve selective detection of VOCs, because the reaction energy of the VOCs molecules is relatively close. Moreover, in order to ionize the surface adsorbed oxygen, MOS sensor requires a higher operating temperature $\left(200^{\circ} \mathrm{C}-500{ }^{\circ} \mathrm{C}\right)$. There is usually a lack of specific reactions to give the sensor selectivity for a certain VOC, either by doping a catalytic reaction, increasing the reaction rate of a certain VOCs, improving selectivity, or improving the selectivity by improving the diffusion rate of a certain VOCs in the material [11-13].

Polymers are a class of highly probable gas-sensitive materials. VOCs have different diffusion coefficients in the polymer, which helps the sensor to obtain good selectivity. Polymer-based gas sensor arrays and electronic noses have achieved good results in detecting VOCs [14]. The polymer can be directly modified on the surface of QCM [15], SAW [16], and SPR devices [17] as a sensitive material; if a conventional resistive gas sensor is to be constructed, the polymer needs to be electrically conductive. For polymers without conductivity, the conductor can be combined with the polymer to make the composite as conductive, which can provide the measurable electrical quantity of the sensor. The resistive gas sensor array is easy to pick up signals and constitutes a miniaturized portable electronic nose. Nanomaterials are particularly attractive due to their high surface area to volume ratio, high sensitivity and fast response time.

In this work, four different polymers and multi-walled carbon nanotubes were tested to form a composite sensitive layer in response to VOCs. Materials, device structures, and testing procedures are described, and the relationships between test resistance response, analyte properties, and polymer chemical structure are analyzed. 


\section{Materials and methods}

Materials and characterization.The polymers used in this work were Polyvinylpyrrolidone (PVP), Poly(acrylic acid) (PAA), Polyethylenimine (PEI) and Polyethylene glycol (PEG), purchased from Aladdin. The chemical structures and average relative molecular mass of these polymers are shown in Table 1. The $\mathrm{N}$ atoms and $\mathrm{O}$ atoms in PVP molecular can act as a hydrogen bond acceptor. The carboxyl group of the PAA polymer can combine with $\mathrm{NH} 3$ to form a new chemical bond. Amino functional groups of the PEI molecule can undergo a nucleophilic addition reaction. The PEG chain contains an oxygen atom to form a hydrogen bond [18]. These properties make the gas-sensitive properties of the four polymers different, and the selective identification of VOCs can be achieved by constructing a gas sensor array.

Table 1 Chemical structures and properties of polymers

\begin{tabular}{lcl}
\hline Polymer & Chemical structure & Average molecular weight \\
Polyvinylpyrrolidone (PVP) & & \\
Poly(acrylic acid) (PAA) & & 8000 \\
Polyethylene glycol (PEG) & & 8000 \\
\hline
\end{tabular}

Gas sensor array fabrication. The sensor array contains a number of different gas sensors. As shown in Fig. 1(a), 16 sensor array chips can be processed on a 4-inch silicon wafer. Each sensor array chip contains 10 sensor elements, each sensor element consisting of A stack of interdigitated electrodes (IDE), as shown in Fig. 1(b), detects the resistance of sensitive materials. One end of the IDEs are connected to a common ground line, the other end is individually led to the particular element, and finally bonded to the PCB package by a gold wire, as shown in Fig. 1(c)(d).
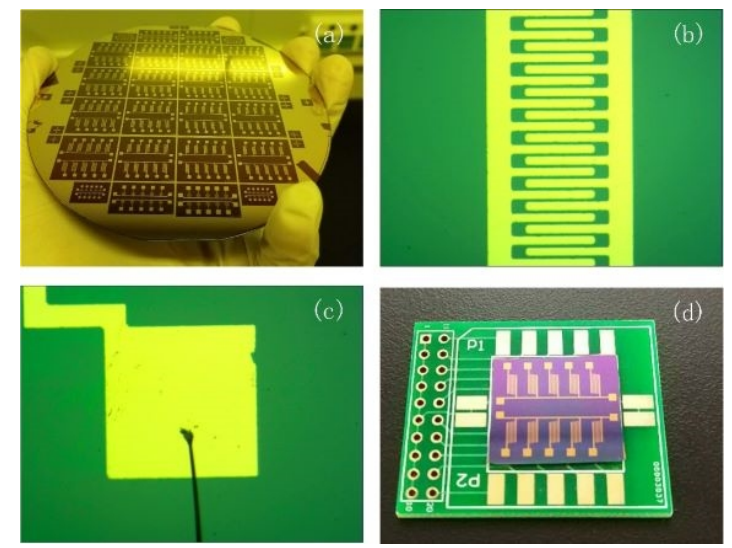

Fig. 1 Photos of sensor array. (a)lift-off results. (b)interdigitated electrode. (c)wire bonding. (d)direct chip attach

The sensor chip is completed on a silicon oxide substrate. The array pattern is first processed on the substrate by photolithography, and the metal is deposited by electron beam, including a $4 \mathrm{~nm} \mathrm{Cr}$ adhesion layer and a $100 \mathrm{~nm}$ gold layer. After the metal layer is deposited, the photoresist is dissolved in an acetone organic solvent, and lift-off is performed to remove excess metal to obtain an electrode 
array. A sensor array chip is obtained after dicing. The wire bonding is performed by a gold wire ball welder to package the chip on the PCB.

Gas sensing experiments. The experimental platform is shown in Fig. 2. The dry air supply is split into two gas branches, one is used as the cleaning gas, and the other dry air flow is passed through the analyte bubbler to generate saturated analyte vapor, which is then mixed with the previous dry air. The gas flow rate of the two branches is controlled by the MFC to obtain a mixed gas diluted to a specific concentration, and the total flow rate of the mixed gas is controlled at $500 \mathrm{ml} / \mathrm{min}$. The NI Source Unit is powered. The NI DAQ collects the voltage of the sensor array and controls the entire system through a LabVIEW program written on the PC.

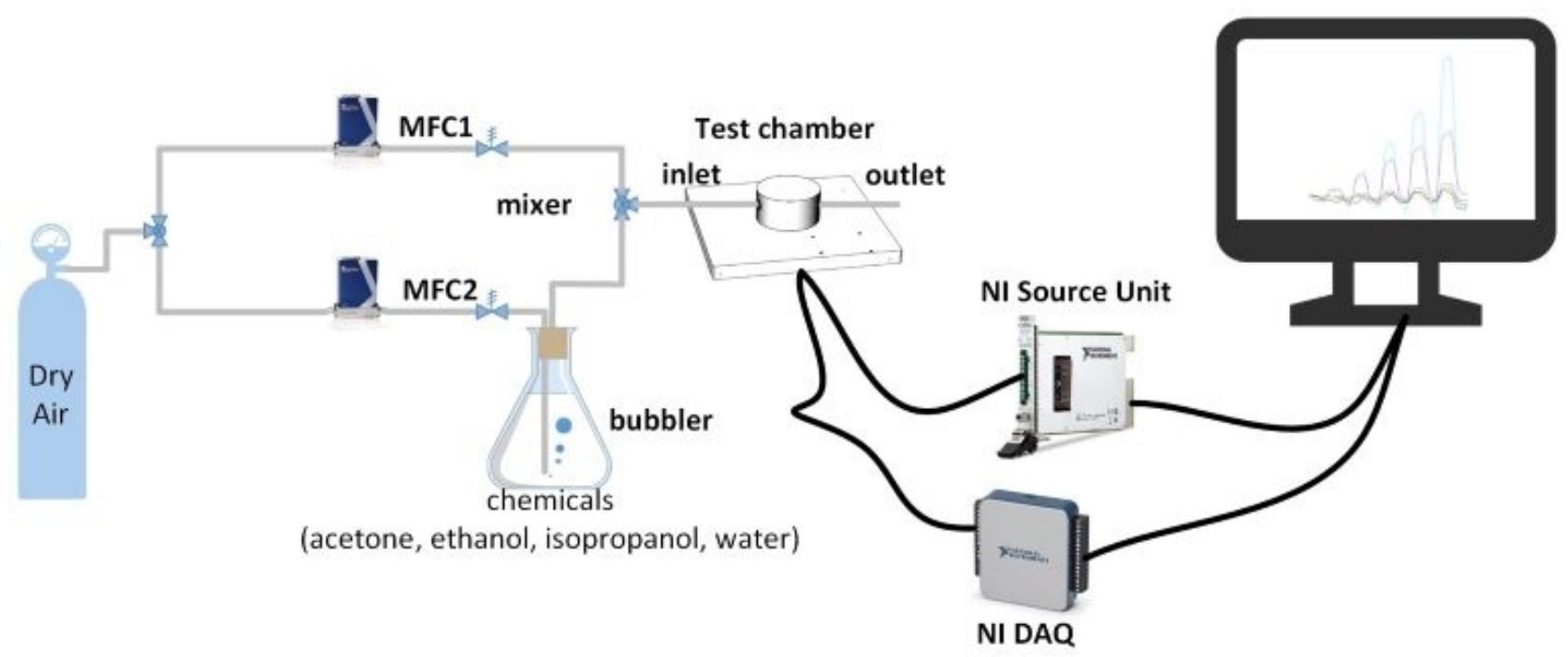

Fig. 2 Schematic diagram of the experimental setup for gas sensing measurement

\section{Results and Discussion}

Materials characterization. The morphology of the obtained sensitive layer is also related to the preparation process. In this study, different sensitive layers in the sensor array are dried by dip-coated MWCNTs dispersion and sprayed polymer solution. The different sensitive layers composed of the MWCNTs and PVP, PAA, PEI, PEG four polymers respectively are shown in Fig. 3(a-d). The drying process of the sensitive layer shows a distinct "coffee ring effect". During the drying process of the droplets, the capillary phenomenon fixes the boundary of the droplet, and the evaporation at the edge is faster than the solvent in the central region of the droplet. The difference results in convective flow within the droplet from the center to the edge (Marangoni effect) [19]. This flow carries the deposit to the edge of the droplet, while the deposited material prevents the edge of the droplet from receding and "fixes" it in its original position. This further promotes the accumulation of material at the edge of the droplet, resulting in a non-uniformity of the sensitive layer and more material deposited at the edge portion, which is commonly referred to as the "coffee ring effect" [20]. Thus the thickness of the sensitive layer above the IDE Thinner, which facilitates the full diffusion of gas molecules in the sensitive layer. 

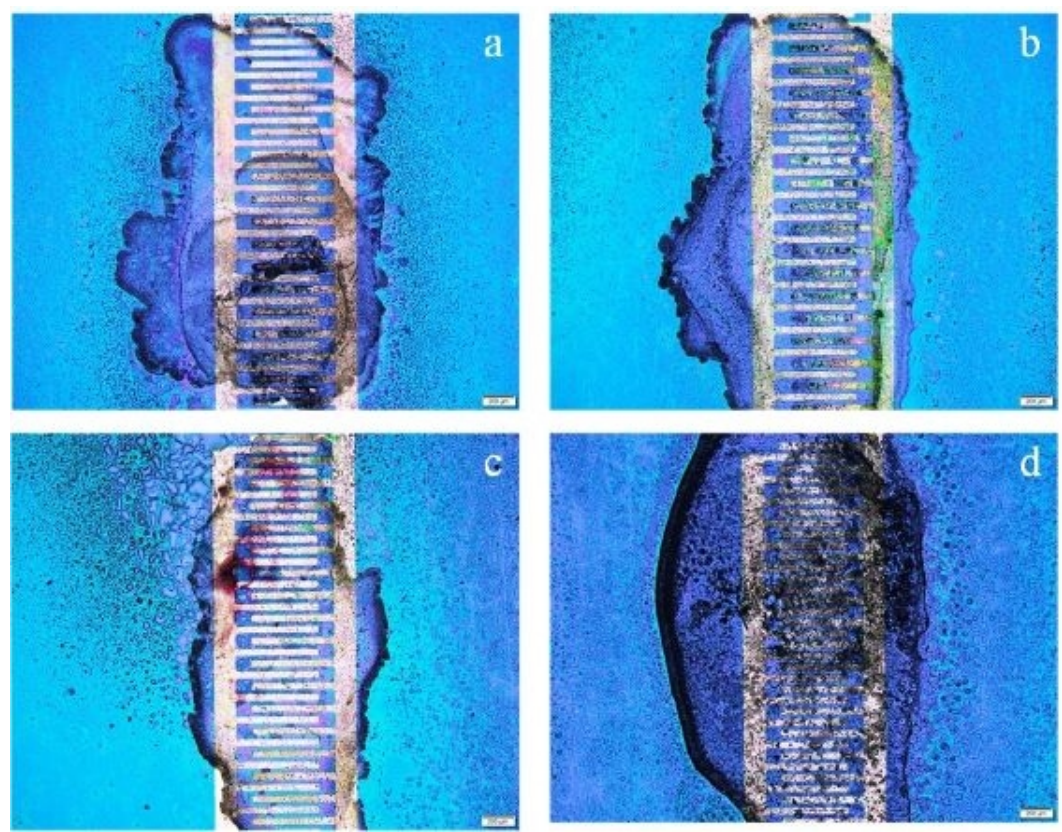

Fig. 3 Optical micrographs of sensitive layer. (a)MWCNTs and PVP. (b)MWCNTs and PAA. (c)MWCNTs and PEI. (d)MWCNTs and PEG

Sensor array response. The response of the sensor array to different concentrations of acetone, ethanol, isopropanol and water is shown in Fig. 4. The resistance of the sensors of different sensitive materials is not the same. The relative change of the resistance of the sensor is calculated as a response signal for comparison. The sensitivity of the composite sensitive layer with PVP and PEG is higher; the sensor based on MWCNTs and PVP composite is more sensitive to ethanol and isopropanol; the response speeds of different gases are different, and the drift directions caused by different gases are also inconsistent. These differences can be used as additional Features, through more features to achieve a more detailed distinction between different types of VOCs. 

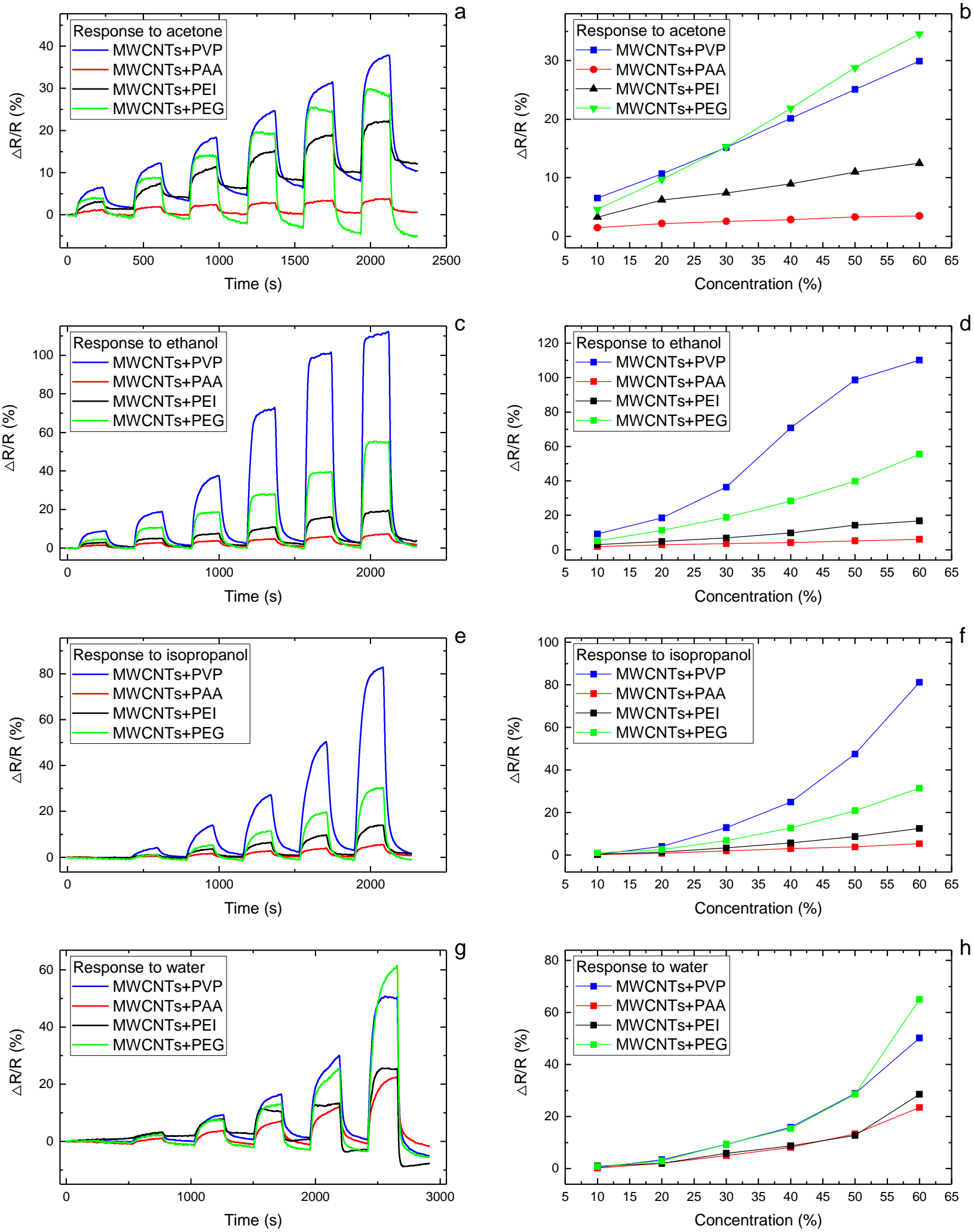

Fig. 4 Sensor responses for various sensitive layer and vapors. The left column of figures is time response curves while the right column of figures shows the response to concentration. The analytes corresponding to the figures are (a-b) acetone, (c-d) ethanol, (e-f) isopropanol, and (g-h) water.

Principal component analysis. The results obtained by principal component analysis are shown in Fig. 5. In the three-dimensional space, acetone, water, isopropanol and ethanol can be distinguished, especially acetone and water have large differences, while ethanol and isopropanol are close in nature. The difference in the figure is not so obvious. It is difficult to distinguish at low concentrations and 
better at high concentrations. The characteristics of acetone and water show a good linear correlation with concentration.

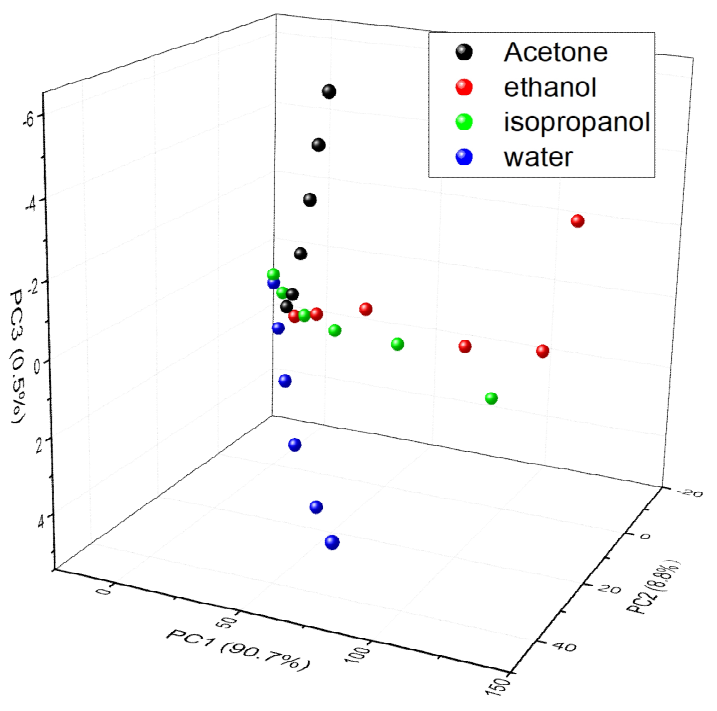

Fig. 5 Principal component score plot of response of sensor array to 4 vapors at six different concentrations

\section{Conclusions}

In conclusion, PVP, PAA, PEI and PEG are combined with MWCNTs to construct a set of resistive gas sensor arrays. VOC molecules diffuse in the sensitive layer, adsorb, affect the conductive network of the sensitive layer, change its resistance and cause sensor response. The experimental results show the sensor's good sensitivity to acetone and ethanol. And the response characteristics of the sensor array are extracted by PCA, which can distinguish acetone, water, ethanol and isopropanol well, which shows the good prospect of the application of sensor array scheme in VOC detection and analysis.

\section{Acknowledgements}

This work is supported by Shenzhen Science and Technology Research and Development Funds (No: JCYJ20150831192244849 and No: JCYJ20160411164305110). The authors also acknowledge the support from the National High Technology Research and Development Plan of China (2015AA043505).

\section{References}

[1] Chang J E, Lee D S, Ban S W, et al. Analysis of volatile organic compounds in exhaled breath for lung cancer diagnosis using a sensor system[J]. Sensors and Actuators B: Chemical, 2018, 255: 800-807.

[2] Gregis G, Sanchez J B, Bezverkhyy I, et al. Detection and quantification of lung cancer biomarkers by a micro-analytical device using a single metal oxide-based gas sensor[J]. Sensors and Actuators B: Chemical, 2018, 255: 391-400.

[3] Wang Z, Wang C. Is breath acetone a biomarker of diabetes? A historical review on breath acetone measurements[J]. Journal of breath research, 2013, 7(3): 037109.

[4] Guo D, Zhang D, Li N, et al. Diabetes identification and classification by means of a breath analysis system[C]//International Conference on Medical Biometrics. Springer Berlin Heidelberg, 2010: 52-63. 
[5] Greiter M B, Keck L, Siegmund T, et al. Differences in exhaled gas profiles between patients with type 2 diabetes and healthy controls[J]. Diabetes technology \& therapeutics, 2010, 12(6): 455-463.

[6] Minh T D C, Oliver S R, Ngo J, et al. Noninvasive measurement of plasma glucose from exhaled breath in healthy and type 1 diabetic subjects[J]. American Journal of Physiology-Endocrinology and Metabolism, 2011, 300(6): E1166-E1175.

[7] Greiter M B, Keck L, Siegmund T, et al. Differences in exhaled gas profiles between patients with type 2 diabetes and healthy controls[J]. Diabetes technology \& therapeutics, 2010, 12(6): 455-463.

[8] Benammar M, Abdaoui A, Ahmad S H M, et al. A Modular IoT Platform for Real-Time Indoor Air Quality Monitoring[J]. Sensors, 2018, 18(2): 581.

[9] Szulczyński B, Gębicki J. Currently commercially available chemical sensors employed for detection of volatile organic compounds in outdoor and indoor air[J]. Environments, 2017, 4(1): 21.

[10] Moseley P T. Progress in the development of semiconducting metal oxide gas sensors: a review[J]. Measurement Science and Technology, 2017, 28(8): 082001.

[11] Güntner A T, Pineau N J, Chie D, et al. Selective sensing of isoprene by Ti-doped ZnO for breath diagnostics[J]. Journal of Materials Chemistry B, 2016, 4(32): 5358-5366.

[12] Güntner A T, Koren V, Chikkadi K, et al. E-nose sensing of low-ppb formaldehyde in gas mixtures at high relative humidity for breath screening of lung cancer?[J]. Acs Sensors, 2016, 1(5): 528-535.

[13] Righettoni M, Tricoli A, Pratsinis S E. Si: WO3 sensors for highly selective detection of acetone for easy diagnosis of diabetes by breath analysis[J]. Analytical chemistry, 2010, 82(9): 3581-3587.

[14] Li B, Santhanam S, Schultz L, et al. Inkjet printed chemical sensor array based on polythiophene conductive polymers[J]. Sensors and Actuators B: Chemical, 2007, 123(2): 651-660.

[15] Nanto H. Odor sensor using plasma-polymerized-film-coated QCM for environmental monitoring $[\mathrm{C}]$. Environmental and Industrial Sensing. International Society for Optics and Photonics, 2002, 4576: 238.

[16] Wang W, He S, Li S, et al. Enhanced sensitivity of SAW gas sensor coated molecularly imprinted polymer incorporating high frequency stability oscillator[J]. Sensors \& Actuators B Chemical, 2007, 125(2):422-427.

[17] Fu D, Chung J, Liu Q, et al. Polymer coated silicon microring device for the detection of sub-ppm volatile organic compounds[J]. Sensors and Actuators B: Chemical, 2018, 257: 136-142.

[18]Zhao B, Hu H, Yu A, et al. Synthesis and characterization of water soluble single-walled carbon nanotube graft copolymers[J]. Journal of the American Chemical Society, 2005, 127(22): 8197-8203.

[19]Zeytounian R K. Theoretical aspects of interfacial phenomena and Marangoni effect[M]//nterfacial Phenomena and the Marangoni Effect. Springer, Vienna, 2002: 123-190.

[20] Singh M, Haverinen H M, Dhagat P, et al. Inkjet printing-process and its applications[J]. Advanced materials, 2010, 22(6): 673-685. 